\title{
Combining Speedup Techniques based on Landmarks and Containers with parallelised pre- processing in Random and Planar Graphs
}

\author{
R. Kalpana ${ }^{1}$ and Dr. P. Thambidurai ${ }^{2}$ \\ ${ }^{1}$ Department of Computer Science \& Engineering, Pondicherry Engineering College, \\ Puducherry, India \\ rkalpana@pec.edu \\ ${ }^{2}$ Perunthalaivar Kamarajar Institute of Engineering \& Technology \\ Karaikal, Puducherry, India
}

\begin{abstract}
The Dijkstra's algorithm is applied in many real world problems like mobile routing, road maps, railway networks, etc,. There are many techniques available to speedup the algorithm while guaranteeing the optimality of the solution. Almost all of the speedup techniques have a substantial amount of parallelism that can be exploited to decrease its running time. By suitably modifying portions of the existing system various degrees of parallelism can be achieved. The rapidly growing field of multiprocessing systems and multi-core processors provide many opportunities for such improvements. In these techniques there's always a demand for the running time and the time required for pre-processing. Space requirements for the pre-processing also have a major influence on the running time of the algorithm.

The main focus of the work is to implement landmark technique and to identify the segment of the code in landmark pre-processing which can be parallelized to obtain better speedup. The results are applied to the combined speedup technique which is based on landmarks and containers. The experimental results were compared and analysed for determining better performance improvements in random graphs and planar graphs.
\end{abstract}

\section{KEYWORDS}

Dijkstra's Algorithm, Graph Theory, parallel execution, speed-up

\section{INTRODUCTION}

A directed simple graph $\mathrm{G}$ is a pair $(\mathrm{V}, \mathrm{E})$, where $\mathrm{V}$ is the set of nodes / vertices and $E \subseteq V \times V$ is a set of edges, where an edge is an ordered pair of nodes of the form (u, v) such that $u, v \in V$. Usually the number of nodes $|V|$ is denoted by $\mathrm{n}$ and the number of edges $|E|$ is denoted by $\mathrm{m}$. A path in graph $\mathrm{G}$ is a sequence of nodes $\left(u_{1}, \ldots, u_{k}\right)$ so that $\left(u_{i}, u_{i+1}\right) \in E$ for all $1 \leq i<k$. A path in which $u_{1}=u_{k}$ is called a cycle or cyclic path.

Given the edge weights $l: E \rightarrow R$, the length of the path $P=\left(u_{1}, \ldots, u_{k}\right)$ is the sum of the lengths of its edges $l(P):=\Sigma_{1 \leq i<k} l\left(u_{i}, u_{i+1}\right)$. For any two nodes $s, t \in V$, a shortest s-t path is a path of minimal length with $u_{1}=s$ and $u_{k}=t$. The distance $d(s, t)$ between $\mathrm{s}$ and $\mathrm{t}$ is the length of the shortest path s-t. A layout of a graph $G=(V, E)$ is a function $L: V \rightarrow R^{2}$ that assigns each node a position in $R^{2}$. A graph is called sparse if $m=O(n)$.

Let $\mathrm{G}=(\mathrm{V}, \mathrm{E})$ be a directed graph whose edges are weighted by a function $w: E \rightarrow R$. The weights are interpreted as the edges' or lengths in the sense that the length of a path is the sum of the weights of its edges. The single-source single-target (SSST) shortest-path problem 
consists in finding a path of minimum length from a given source $s \in V$ to a given target $t \in V$. The problem is only well defined for all pairs, if $\mathrm{G}$ does not contain negative cycles. In the presence of negative weights, but not negative cycles, it is possible, using Johnson's algorithm, to convert in $O\left(n m+n^{2} \log n\right)$ time the original edge weights $w: E \rightarrow R$ to non-negative edge weights $w^{\prime}: E \rightarrow R_{0}{ }^{+}$that result in the same shortest paths. Hence, it can be safely assumed that the edge weights are non-negative. It can also be assumed that for all pairs $(s, t) \in V \times V$, the shortest path from $s$ to $t$ is unique.

The classical algorithm for computing shortest paths in a directed graph with nonnegative edge weights is that of Dijkstra's algorithm. Dijkstra's algorithm implemented with Fibonacci heaps is still the fastest known algorithm for the general case of arbitrary nonnegative edge lengths, taking $O(m+n \log n)$ worst-case time. For special cases (eg. undirected graphs, integral or uniformly distributed edge weights), better algorithms are identified.

\section{RELATED WORK}

\subsection{Basic speedup techniques}

Computing shortest paths between nodes in a given directed graph is classically solved by Dijkstra's algorithm[1]. But besides Dijkstra's algorithm there are many recent algorithms that solve variants and special cases of the shortest-path problem with better running time. This section also focuses on variants of Dijkstra's algorithm (also denoted as speedup techniques in the following) that further exploit the fact that a target is given. Typically, such improvements of Dijkstra's algorithm cannot be proved to be asymptotically faster than the original algorithm, and, in this sense are heuristics. However, it can be empirically shown that they indeed improve the running time drastically for many realistic data sets. An overview of the speedup techniques is as follows

- Goal-directed search: The given edge weights are modified to favour edges leading toward the target node [2]. With graphs from timetable information, a speed-up in running time of a factor of roughly 1.5 is reported [2]

- Bidirectional search: Start a second search backward, from the target to the source. Both searches[3] stop when their search horizons meet. Using bidirectional search space can be reduced by a factor of 2 .

- Multilevel approach: This approach takes advantage of hierarchical coarsening of the given graph, where additional edges have to be computed. These edges can be regarded as distributed to multiple levels. Depending on the given query, only a small fraction of these edges have to be considered to find a shortest path. Using this technique, speed-up factors of more than 3.5 were observed for road map and public transport graphs [4]. Timetable information queries could be improved by a factor of 11 [5].

- Shortest-path containers: These containers[6] provide a necessary condition for each edge, whether or not it has to be respected during the search. More precisely, the set of all nodes that can be reached on a shortest path using this edge is stored. Speedup factors in the range between 10 and 20 can be achieved.

\subsection{Combining Speedup Techniques}

The key notion of combining each pair of techniques is outlined[7] and it is noted that extending these to combinations, including three or all four techniques, are not difficult.

Goal-Directed Search and Bidirectional Search. Combining goal-directed and bidirectional search is not as obvious as it may seem. Simple application of a goal-directed search forward and backward yields a wrong termination condition. In certain situations the 
search in each direction almost reaches the sources of the other direction. This often results in a slower algorithm.

To overcome these deficiencies, it is preferable to use the very same edge weights $l^{\prime}(v, w)=l(v, w)-\lambda(v)+\lambda(w)$ for both the forward and the backward search. With these weights, the forward search is directed to the target $t$ and the backward search has no preferred direction, but favours edges that are directed towards $t$. This proceeding always computes shortest paths, as an $s-t$ path is shortest independent of whether $l$ or $l^{\prime}$ is used for the edge weights.

Goal-Directed Search and Multilevel Approach. The multilevel approach determines, for each query, a subgraph of the multilevel graph on which Dijikstra's algorithm is finally run. The computation of this subgraph does not affect edge lengths and thus a goal-directed search can be simply performed on it.

Goal-Directed Search and Shortest-Path Containers. Similar to the multilevel approach, the shortest-path containers approach determines for a given query a subgraph of the original graph. Again, edge lengths are irrelevant for the computation of the subgraph and goal-directed search can be applied readily. Even though the Euclidian distances were combined with containers[ ] the landmarks are not considered for containers yet.

Bidirectional Search and Multilevel Approach. A bidirectional search can be applied to the subgraph defined by the multilevel approach. The subgraph can be computed on the fly during Dijikstra's algorithm: for each node considered, the set of necessary outgoing edges is determined. To perform a bidirectional search on the multilevel subgraph, a symmetric, backward version of the subgraph computation has to be implemented: for each node considered in the backward search, the incoming edges that are part of the subgraph have to be determined. Shortest paths are guaranteed, since bidirectional search is run on a subgraph that preserves optimality, and, by the additional edges, only contains supplementary information consistent with the original graph.

Bidirectional Search and Shortest-Path Containers. In order to take advantage of shortest-path containers in both directions of a bidirectional search a second set of containers is needed. For each edge e $\in \mathrm{E}$, the set $\mathrm{S}_{\mathrm{b}}(\mathrm{e})$ is computed containing those nodes from which a shortest path ending with e exists. For each edge e $\in \mathrm{E}$ the bounding box of $\mathrm{S}_{\mathrm{b}}(\mathrm{e})$ is stored in an associative array $\mathrm{C}_{\mathrm{b}}$ with index set $\mathrm{E}$. The forward search checks whether the target is contained in $\mathrm{C}(\mathrm{e})$, the backward search, checks whether the source is in $\mathrm{C}_{\mathrm{b}}(\mathrm{e})$. It can be verified that by construction only such edges are pruned that do not form part of any partial shortest path and thus of any shortest $s$ - $t$ path.

Multilevel Approach and Shortest-Path containers. The multilevel approach enriches a given graph with additional edges. Each new edge $\left(\mathrm{u}_{1}, \mathrm{u}_{\mathrm{k}}\right)$ represents a shortest path $\left(\mathrm{u}_{1}, \mathrm{u}_{2}, \ldots, \mathrm{u}_{\mathrm{k}}\right)$ in $G$. Such a new edge $\left(\mathrm{u}_{1}, \mathrm{u}_{\mathrm{k}}\right)$ is annotated with $C\left(\mathrm{u}_{1}, \mathrm{u}_{2}\right)$, the associated bounding box of the first edge on this path. This consistent labelling of new edges, which represent shortcuts in the original graph, ensures still shortest paths.

Hierarchical and Goal-directed speed-up techniques. The combination of hierarchical and goal directed speedup techniques [8], [9] found to give best results for unit disk graphs, grid networks, and time-expanded timetables. It is suggested that the goal directed technique can be applied to higher levels of hierarchy.

\section{$2.3 \mathrm{~A} *$ Search and Landmarks}

In this section a new shortest path algorithm that uses $\mathrm{A}^{*}$ search in combination with a new graph-theoretic lower-bounding technique based on landmarks and the triangle inequality is explained[8]. The algorithm computes optimal shortest paths and works on any directed graph. Experimental results show that the new technique outperforms $A^{*}$ search with Euclidean bounds, by a wide margin on road networks [2].

\section{Potential Function[8]}


A potential function is a function, from vertices to reals. Given a potential function $\pi$, the reduced cost of an edge is defined as follows

$$
l_{\pi}(v, w)=l(v, w)-\pi(v)+\pi(w)
$$

Suppose $l$ is replaced by $l_{\pi}$ then for any two vertices $\mathrm{x}$ and $\mathrm{y}$, the length of any $\mathrm{x}$-y path changes by the same amount $\pi(y)-\pi(x)$. Thus a path is a shortest path with respect to $l$ iff it is a shortest path with respect to $l_{\pi}$ and the two problems are equivalent. Note that, $\pi$ is feasible if $l_{\pi}$ is nonnegative for all arcs. It is well known that if $\pi(t) \leq 0$ and $\pi$ is feasible, then for any $\mathrm{v}, \pi(v)$ is a lower bound on the distance from $\mathrm{v}$ to $\mathrm{t}$. It is to be noted that

Lemma 2.1 If $\pi_{1}$ and $\pi_{2}$ are feasible potential functions, then $p=\max \left(\pi_{1}, \pi_{2}\right)$ is feasible.

Feasible potential functions can be combined by taking the minimum, or, the average of feasible potential functions. The maximum is used in particular to combine feasible lower bound functions in order to get one that any vertex is at least as high as each original function.

A* Search[8]

Consider the problem of looking for a path from $s$ to $t$ and assume that a function $\pi_{t}: V \rightarrow R$ exists such that $\pi_{t}(v)$ gives an estimate on the distance from $\mathrm{v}$ to $\mathrm{t}$. $\mathrm{A}^{*}$ search is an algorithm that works like Dijkstra's algorithm, except that at each step it selects a labelled vertex $\mathrm{v}$ with the smallest value $k(v)=d_{s}(v)+\pi_{t}(v)$ to scan next. It is easy to verify that $\mathrm{A}^{*}$ search is equivalent to Dijkstra's algorithm on the graph with length function ${ }^{l} \pi_{t}$.

\subsection{Geometric containers for efficient shortest path computation}

A fundamental approach in finding efficiently best routes or optimal itineraries in traffic information is to reduce the search space of the most commonly used shortest path routine (Dijikstra's algorithm) on a suitable defined graph. Reduction of the search space should simultaneously be combined with ways of retaining data structures, created during a preprocessing phase of size linear to the size of the graph. The search space of Dijikstra's algorithm can be significantly reduced by extracting geometric information from a given layout of the graph and by encapsulating precomputed shortest-path information in resulted geometric objects (containers) [6]. When edge weights are subject to change, methods exist for dynamically updating the containers instead of recomputing everything from scratch [6].

\subsubsection{Shortest-Path Containers}

In this section, we consider the concept of containers, which helps to reduce the search space of Dijkstra's algorithm. Containers are used to keep the nodes, which are potentially useful for shortest-path computations. This idea gives rise to Dijkstra's Algorithm with Pruning [6], which reduces the search space by examining, at each iteration, only a subset of the neighbors of a node (line 5a); the differences to Dijkstra's algorithm are shown in boldface. The condition in line $5 \mathrm{a}$ is formalized by the notion of a consistent container[6].

\subsection{OpenMP}

OpenMP is an Application Programming Interface (API) [11] for portable shared memory parallel programming. The API allows an incremental approach to parallelize an existing code, so that portions of a program can be parallelized in successive steps. This is a marked difference between OpenMP and other parallel programming paradigms where the entire program must be converted in one step. 


\subsubsection{Fork and Join Model of OpenMP}

Multithreaded programs can be written in different ways that allows complex interaction between threads. OpenMP provides a simple structured approach to multithreaded programming. OpenMP supports a fork-join programming model as shown in figure 1.

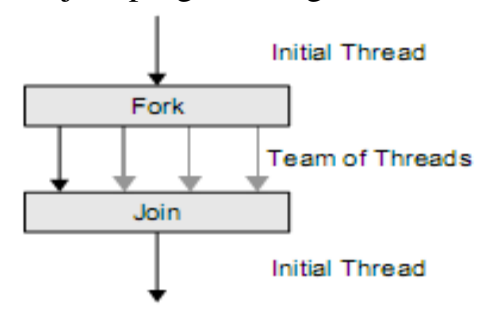

Figure 1. Fork-Join programming model of OpenMP

In this approach, the program starts as a single thread of execution. Whenever an OpenMP parallel construct is encountered by a thread while it is executing the program, it creates a team of threads (this is the fork), becomes the master of the team, and collaborates with the other members of the team to execute the code enclosed by the construct. At the end of the construct, only the original thread continues; all others terminate (this is the join). Each portion of code enclosed by a parallel construct is called a parallel region. OpenMP expects the application developer to give a high-level specification of the parallelism in the program and the method for exploiting that parallelism. The OpenMP implementation will take care of the lowlevel details of actually creating independent threads to execute the code and to assign work to them according to the strategy specified by the programmer. The shortest path problem is solved in parallel with OpenMP in [10]. Although there is improvement in speedup, there are many speedup techniques which can be parallelized and the performance can be improved.

\subsubsection{Amdahl's Law}

If $T 1$ denotes the execution time of an application on 1 processor, then in an ideal situation, the execution time on $P$ processors should be $T 1 / P$. If $T P$ denotes the execution time on $P$ processors, then the ratio

$$
S=T_{1} / T_{P}
$$

is referred to as the parallel speedup and is a measure for the success of the parallelization. However, a number of obstacles usually have to be overcome before perfect speedup is achievable. Virtually all programs contain some regions that are suitable for parallelization and other regions that are not. By using an increasing number of processors, the time spent in the parallelized parts of the program is reduced, but the sequential section remains the same. Eventually the execution time is completely dominated by the time taken to compute the sequential portion, which puts an upper limit on the expected speedup. This effect, known as Amdahl's law, can be formulated as

$$
S=\frac{1}{\left(f_{\text {par }} / P+\left(1-f_{\text {par }}\right)\right)}
$$

where fpar is the parallel fraction of the code and $P$ is the number of processors. In the ideal case when all of the code runs in parallel, fpar $=1$, the expected speedup is equal to the number of processors.

\section{SPEEDUP TECHNIQUES WITH AND WITHOUT PARALLELISM}

\subsection{LANDMARKS}

The search space of Dijkstra's algorithm can be reduced by using landmarks. Heuristic estimates on the distance of a vertex to the target can be calculated using landmarks. Landmarks 
tend to attract the search towards them and so by appropriately selecting landmarks the overall performance can be improved.

The procedure in Algorithm 1 outlines the shortest path computation technique with heuristic values modifying the priority of vertices. Lines $4 a$ and 6 are the changes made to the original Dijkstra's algorithm. The purpose of line $4 \mathrm{a}$ is evident of its own because the problem under consideration is single source single target shortest path problem. The key change is that of line 6. Traditional Dijkstra's algorithm considers only the distance of a vertex from the source whereas in Algorithm 1 potential(u) is used an estimate of the distance from the vertex to the target. So a heuristic / potential function can direct the search towards the target thereby reducing the search space considerably.

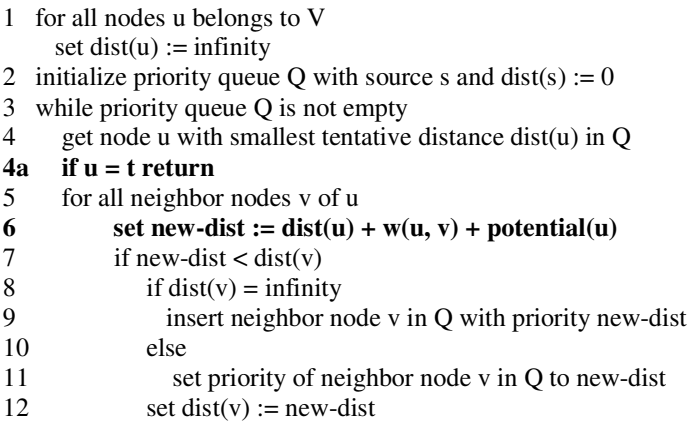

Algorithm 1. Using Landmarks in Shortest Path Computation

\subsubsection{Landmark Selection}

For incorporating landmarks into shortest path computation the following additions are to be made to the existing path computation technique: A procedure for selecting landmarks, computing the distance values from the landmarks to the remaining vertices and utilizing the computed distance values to obtain heuristic estimates which could be used to modify the priority of vertices to be considered.

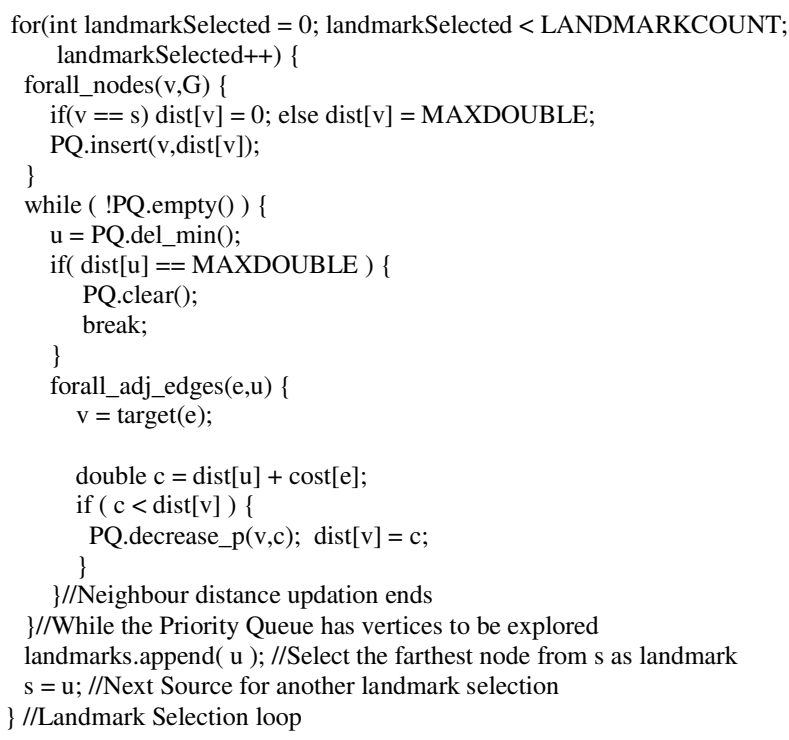

Code Segment 1. Farthest Landmark Selection Technique

The landmark selection procedure is briefed in code segment 1 . The procedure used for selecting landmarks is called "Farthest landmark selection technique". The idea behind this 
procedure is that, landmarks are chosen in such a way that they are far apart, i.e. the landmarks are spread throughout the entire graph and this helps to obtain good potential values for any vertex chosen at random without any bias. The data structure "landmarks" is a list containing the landmarks chosen by the procedure. "LANDMARKCOUNT" indicates the number of landmarks required.

The selection procedure proceeds as follows. A single source all target shortest path query is initiated similar to traditional Dijkstra's algorithm. The vertices deleted from the priority queue are kept track of and the final vertex to be deleted from the queue is added to the list of landmarks. The final vertex is selected as a landmark because in Dijkstra's algorithm the vertices are always considered in the increasing order of their shortest path distance and the final vertex deleted from the queue is the one farthest from the source. The selection procedure is repeated with the newly selected landmark as the source. Once the required number of landmarks are selected the procedure stops.

\subsubsection{Calculating and Using Heuristic Values}

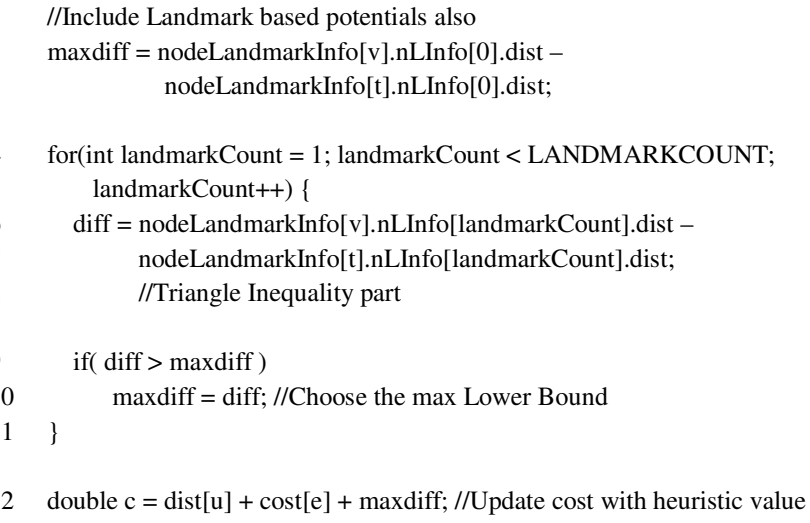

Code Segment 2. Updating cost using Landmark based heuristic values

The distance from landmarks to the remaining vertices should be calculated for obtaining potential values. The distance calculation requires initiating a single source all target shortest path computation from each of the landmarks. The obtained values are stored as follows. "nodeLandmarkInfo" is a vertex array, containing an array of landmark and corresponding distance values. So the distance between a vertex ' $\mathrm{v}$ ' and the $\mathrm{i}^{\text {th }}$ landmark can be accessed as nodeLandmarkInfo[v].nLInfo[i].dist.

Code Segment 2. highlights the modifications to be made during the actual shortest path evaluation. Before updating the distance value of a vertex ' $v$ ', the maximum "distance difference" between the vertex ' $\mathrm{v}$ ' and the various landmarks is calculated and stored in "maxdiff". This serves as a heuristic estimate of the distance between the vertex and the target. Hence the priority is updated only if the sum of, known distance from source and an estimate of the distance from the vertex to the target is less than the previously available priority.

\subsection{SHORTEST PATH CONTAINERS}

The Geometric containers help to reduce the search space of Dijkstra's algorithm by enclosing a list of target nodes for each edge inside a geometric object. The geometric information associated with each edge is then used for improving the performance of shortest path computations. Let $\mathrm{G}=(\mathrm{V}, \mathrm{E}), \mathrm{w}: \mathrm{E} \rightarrow \mathrm{R}$ be a weighted graph. It is remembered that a set of nodes $\mathrm{C} \subseteq \mathrm{V}$ is called a container. A container $\mathrm{C}$ associated with an edge $(\mathrm{u}, \mathrm{v})$ is called consistent, if for all shortest paths from $u$ to $t$ that start with the edge $(\mathrm{u}, \mathrm{v})$, the target $\mathrm{t}$ is in $\mathrm{C}$. 
In other words, $\mathrm{C}(\mathrm{u}, \mathrm{v})$ is consistent, if $\mathrm{S}(\mathrm{u}, \mathrm{v}) \subseteq \mathrm{C}(\mathrm{u}, \mathrm{v})$, where $\mathrm{S}(\mathrm{u}, \mathrm{v})$ represents the set of nodes $\mathrm{x}$ for which the shortest $\mathrm{u}$-x-path starts with the edge $(\mathrm{u}, \mathrm{v})$. Note that further nodes may be part of a consistent container[6]. However, at least the nodes that can be reached by a shortest path starting with $(\mathrm{u}, \mathrm{v})$ must be in $\mathrm{C}(\mathrm{u}, \mathrm{v})$. The additional nodes are referred as wrong nodes, since they lead the search in the wrong way.

\subsubsection{Creating Consistent Containers}

$\mathrm{S}(\mathrm{e})$ is the set of all nodes $\mathrm{t}$ with the property that there is a unique shortest s-t path that starts with the edge e. To determine $S(s, x)$ for every edge $(s, x) \in E$, dijkstra's algorithm is run for every node $s \in V$. A node array "na" is used such that the entry na[v], $v \in V$, stores the first edge ( $\mathrm{s}, \mathrm{x})$ in a shortest s-v path in G. This is constructed in a way similar to the shortest path tree: every time the distance label of a node $\mathrm{v}$ is adjusted via $(\mathrm{u}, \mathrm{v})$, we set na[v] to $(\mathrm{u}, \mathrm{v})$ if $\mathrm{u}=\mathrm{s}$ and to $\mathrm{na}[\mathrm{u}]$ otherwise (Lines $11-14$ of code segment 3 ).

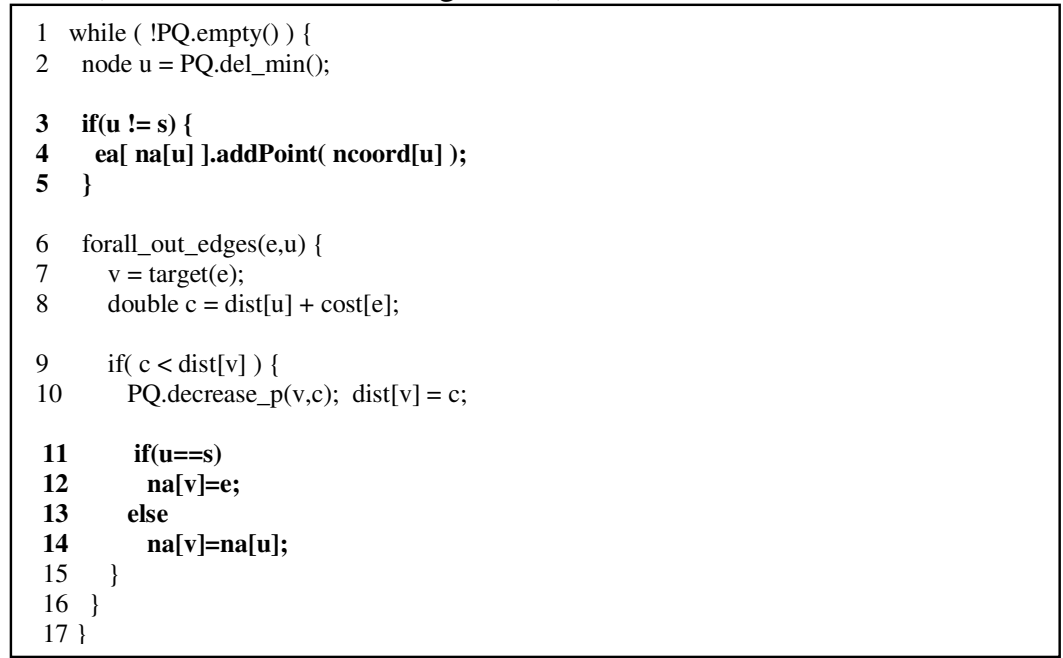

Code Segment 3. Container Construction

When a node $\mathrm{u}$ is removed from the priority queue PQ, na[u] holds the outgoing edge with which a shortest path from $\mathrm{s}$ to u starts. This information is stored in an edge array "ea". Line 4 invokes the container update routine for associating the vertex ' $u$ ' with the appropriate edge.

The problem that arises from using $\mathrm{S}$ (e) is the space requirements. Storing with each edge, a list of target nodes that can be reached using it would require $\mathrm{O}(\mathrm{mn})$ space where number of edges is $\mathrm{m}$ and the number of nodes is $\mathrm{n}$; this is substantially large for a sparse graph.

Using geometric objects (geometric containers) the space required for storing preprocessed information can be reduced. The impact of using the containers to speedup Dijkstra's algorithm does depend on the relation of layout and edge weights. The containers are best suited for constant graphs because for dynamic graphs where the edge weights change rapidly results in updating the containers which is a costly operation and it requires more time. A container can have wrong nodes. These wrong nodes get naturally added up when the targets associated with a particular edge are far apart in the original layout of the graph.

\subsubsection{Bounding Box}

The geometric object used for testing this speedup technique is the bounding box[6] shown in Figure 2. It suffices to store four numbers for each object, which are the lower, upper, left and right boundary of the box. The bounding boxes can easily be computed online while the shortest paths are computed in the pre-processing. 


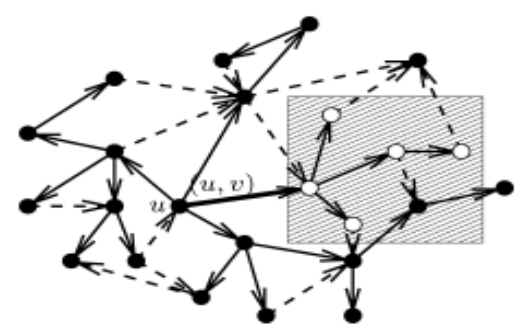

Figure 2. Bounding Box

\section{Expansion of Bounding Box}

The operations involved in computing shortest paths using geometric containers are creating consistent container, enlarging the container associated with each edge and then checking containment of a node within the bounding box while computing the shortest path. The steps involved in creating consistent containers are given in code segment 3 . Enlarging the container for each edge to include the target nodes is performed in code segment 4 . Each node is associated with a coordinate value obtained from the layout of the given graph. If a new vertex is to be added to the container associated with an edge, the co-ordinate values of the new vertex is compared with the existing boundary co-ordinates. The co-ordinates of the containers are adjusted if necessary to include the newly added vertex.

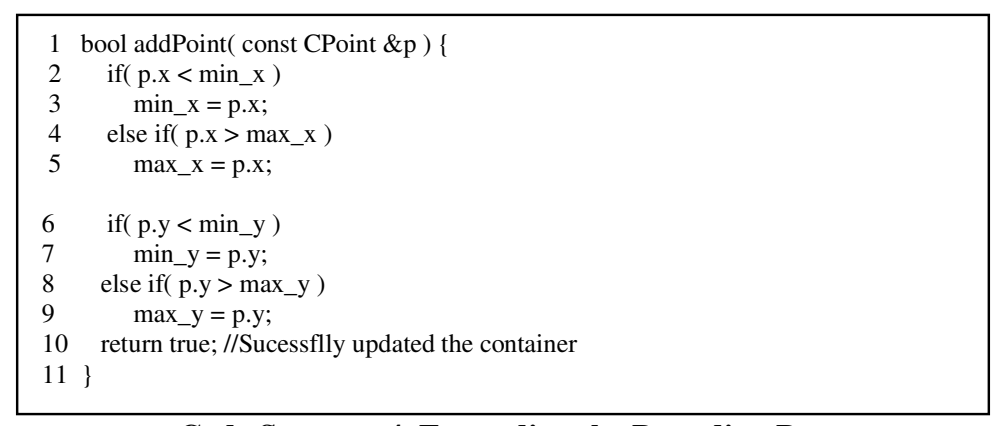

Code Segment 4. Expanding the Bounding Box

While computing the shortest path, when an edge e is reached, the boundary values of that edge $\mathrm{e}$ is checked to see if it contains the target node. If the target is present in the container then the edge is selected otherwise the edge is discarded. Code fragment 5 checks if a given node (specifically if the target) is present in a container.

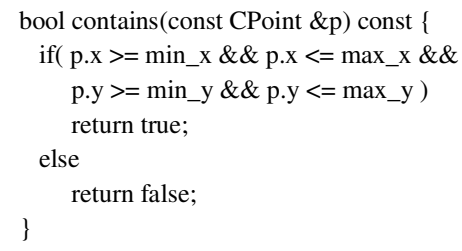

Code Segment 5. Checking the Container 


\subsection{COMBINATION OF LANDMARKS AND GEOMETRIC CONTAINERS}

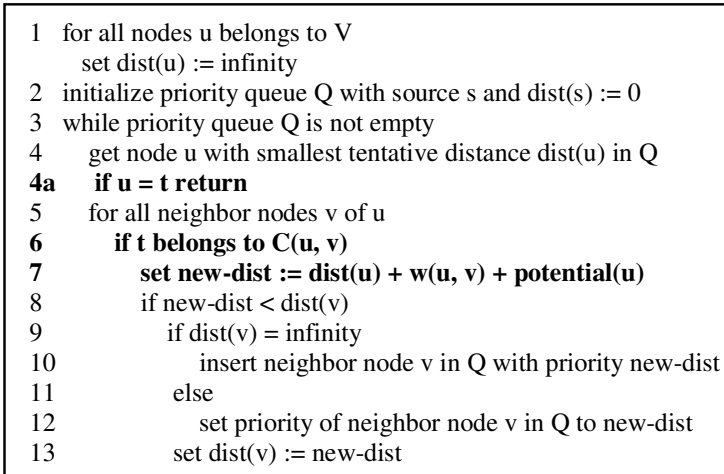

Algorithm 2. Combination of Landmarks and Geometric Containers

The shortest path computation technique that combines both the landmarks and geometric containers is given in Algorithm 2. The changes made to the traditional Dijkstra's algorithm are in lines 4a, 6 and 7. Line 4a terminates the search procedure once the target is reached. Line 6 utilizes the containers for checking if an edge will eventually lead to the specified target. Line 7 in the algorithm uses potential values obtained from landmarks to modify vertex priority.

Line 6 assumes the existence of such a container for its functioning. It is remembered that the container associated with an edge, gives details pertaining to the targets that are reachable, with this edge included in their shortest path. Line 7 uses heuristic values to orient the search towards the target.The benefits of both the containers and landmarks are coupled as follows. The vertex ' $u$ ' to be visited next is deleted from the priority queue in line 4 . The main modification occurs in the neighbour distance updation logic. Traditional Dijkstra's algorithm considers all the neighbours ' $v$ ' of the selected vertex ' $u$ '; using containers only a subset of the neighbours ' $v$ ' to be visited are considered (line 6 of the algorithm), thereby reducing the search space. Then for the selected neighbours ' $\mathrm{v}$ ' the distance to be updated includes an estimate of the distance from the vertex to the target (line 7 of the algorithm) and this helps to focus the search towards the target.

\subsection{Land marks with parallelised preprocessing}

The distance calculation part in the preprocessing phase can be parallelised as follows: The shortest path distance computation between each landmark and the remaining vertices can be carried out in parallel. Code Segment 6 highlights the modifications to be made during the actual shortest path evaluation. Before updating the distance value of a vertex ' $v$ ', the maximum "distance difference" between the vertex ' $v$ ' and the various landmarks is calculated and stored in "maxdiff". This serves as a heuristic estimate of the distance between the vertex and the target. Hence the priority is updated only if the sum of, known distance from source and an estimate of the distance from the vertex to the target is less than the previously available priority. 


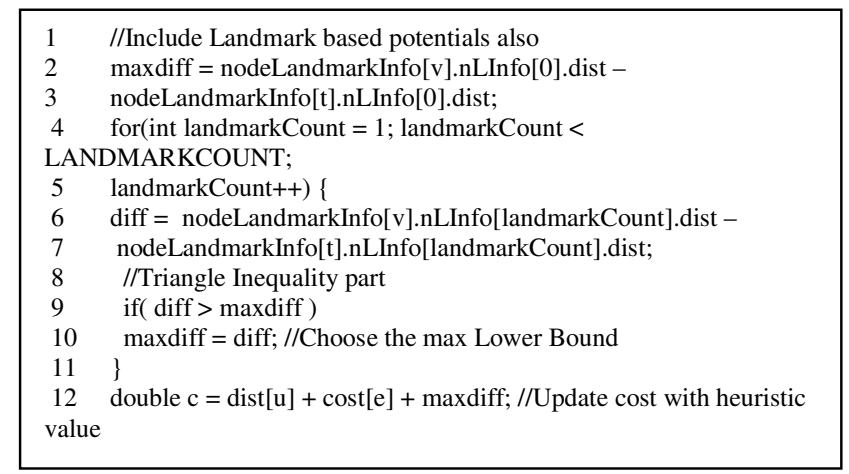

\section{Code Segment 6 Updating cost using Landmark based}

\section{EXPERIMENTAL ANALYSIS}

The different speedup techniques for Dijkstra's algorithm were implemented in C++ with the help of LEDA library version 6.2 (Library of Efficient Data Types and Algorithms) [11]. The graph and priority queue data structures as well as other utilities such precise time measurement function provided by LEDA were used in the implementation. For parallelising the regions of code, the OpenMP API was utilized. The use of OpenMP helps to maintain a single source for both the sequential and parallel versions of the algorithm. The code was compiled using Microsoft ${ }^{\circledR}$ 32-bit C/C++ Compiler (version 15.00.30729.01) and the experiments were performed on an Intel Core2Duo machine $(2.20 \mathrm{GHz})$ with $1 \mathrm{~GB}$ RAM running Windows 7 32-bit operating system.

All the speedup techniques were coded as separate functions, for instance, the bidirectional search and traditional Dijkstra's algorithm were kept as separate modules. The random and planar graph generators provided by LEDA were used for generating graphs on which the modules were tested. The number of vertices visited during the shortest path computation and runtime were measured and used as metrics for comparing the different speedup techniques. The time required for preprocessing and shortest path computation was accurately measured by using the functionality offered by LEDA.

\subsection{ANALYSIS OF LANDMARKS ON RANDOM GRAPHS}

Table 1. Comparison of traditional Dijkstra's algorithm with Landmarks based on running time and vertices visited during shortest path computation on random graphs

\begin{tabular}{|c|c|c|c|c|c|c|}
\hline $\begin{array}{c}\text { Vertex } \\
\text { Count }\end{array}$ & $\begin{array}{c}\text { Edge } \\
\text { Count }\end{array}$ & $\begin{array}{c}\text { Preprocessing } \\
\text { Time (s) }\end{array}$ & $\begin{array}{c}\text { Runtime [with } \\
\text { Landmarks] (s) }\end{array}$ & $\begin{array}{c}\text { Vertices } \\
\text { Visited } \\
\text { [Landmarks] }\end{array}$ & $\begin{array}{c}\text { Runtime } \\
\text { [Dijkstra] (s) }\end{array}$ & $\begin{array}{c}\text { Vertices } \\
\text { Visited } \\
\text { [Dijkstra] }\end{array}$ \\
\hline 10000 & 75500 & 0.332 & 0.0489 & 3698 & 0.0408 & 5365 \\
\hline 11000 & 85250 & 0.41 & 0.0703 & 5215 & 0.0543 & 6295 \\
\hline 12000 & 90600 & 0.477 & 0.0678 & 4626 & 0.0451 & 4739 \\
\hline 13000 & 91000 & 0.518 & 0.081 & 5793 & 0.0536 & 6378 \\
\hline 14000 & 107800 & 0.582 & 0.0759 & 4629 & 0.0621 & 5629 \\
\hline 15000 & 112500 & 0.642 & 0.0899 & 5682 & 0.0715 & 7940 \\
\hline 16000 & 120800 & 0.678 & 0.0984 & 6274 & 0.0739 & 7712 \\
\hline 17000 & 127500 & 0.765 & 0.129 & 7747 & 0.0899 & 9177 \\
\hline 18000 & 133200 & 0.886 & 0.107 & 5814 & 0.105 & 10380 \\
\hline 19000 & 142500 & 0.878 & 0.108 & 6139 & 0.0905 & 8535 \\
\hline 20000 & 161000 & 0.969 & 0.149 & 8147 & 0.132 & 12390 \\
\hline
\end{tabular}




\begin{tabular}{|l|l|l|l|l|l|l|}
\hline 21000 & 155400 & 1.03 & 0.152 & 8313 & 0.125 & 11826 \\
\hline 22000 & 167200 & 1.06 & 0.194 & 11341 & 0.124 & 10558 \\
\hline 23000 & 154100 & 1.04 & 0.189 & 11749 & 0.104 & 10420 \\
\hline 24000 & 166800 & 1.07 & 0.168 & 10432 & 0.119 & 11639 \\
\hline 25000 & 186250 & 1.27 & 0.187 & 10245 & 0.137 & 11857 \\
\hline
\end{tabular}

The following remarks could be made based on the tabulated values. The preprocessing time steadily increases with the number of vertices. This is acceptable because the distance between a landmark and all the remaining vertices are computed during preprocessing. The running time of the modified search procedure with landmarks included is either nearly equal to or slightly higher than that of the traditional Dijkstra's algorithm. The performance with landmarks is expected to improve on real world graphs. The number of vertices visited is reduced by using landmarks. A speedup of nearly 1.2 is achieved. Figure 3 shows the number of vertices visited by the search procedure with landmarks and that of traditional Dijkstra plotted against the number of vertices present in the graph. The number of vertices visited by searching with landmarks is considerably less in most searches.

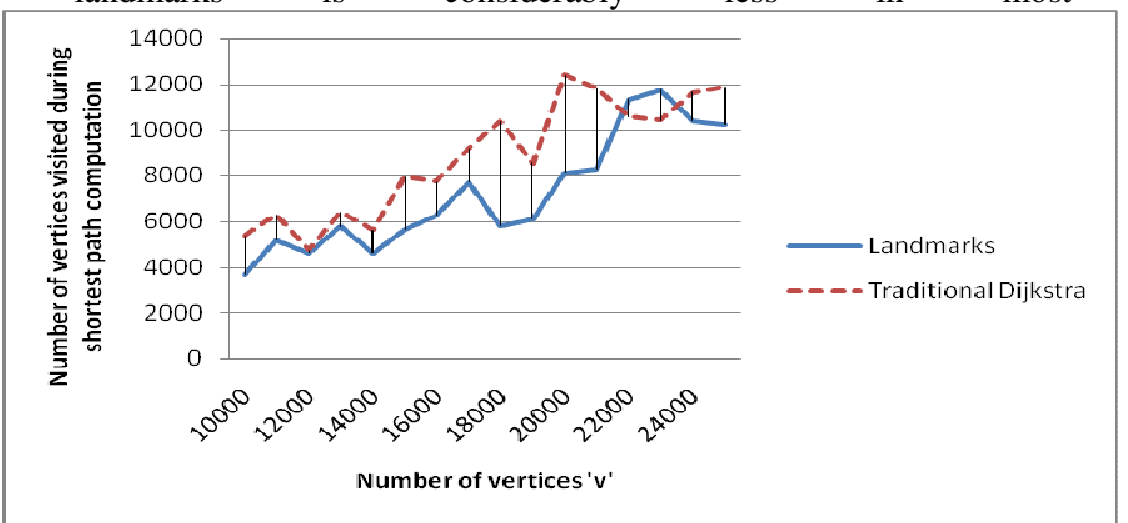

Figure 3. Vertices visited during shortest path computation by traditional Dijkstra and search procedure with Landmarks on random graphs

\subsection{ANALYSIS OF LANDMARKS ON PLANAR GRAPHS}

The effect of using landmarks during shortest path computation on planar graphs is analysed below. The performance of the technique in this graph type is nearly equal to that of traditional Dijkstra's algorithm. Figure 3 plots the vertices visited by traditional Dijkstra and a search using landmarks on the planar graphs generated by LEDA. The values are tabulated in Table 2.

Table 2. Comparison of traditional Dijkstra's algorithm with Landmarks based on running time and vertices visited during shortest path computation on planar graphs

\begin{tabular}{|r|r|r|r|r|r|r|}
\hline $\begin{array}{c}\text { Vertex } \\
\text { Count }\end{array}$ & $\begin{array}{c}\text { Edge } \\
\text { Count }\end{array}$ & $\begin{array}{c}\text { Preprocessing } \\
\text { Time (s) }\end{array}$ & $\begin{array}{c}\text { Runtime [with } \\
\text { Landmarks] (s) }\end{array}$ & $\begin{array}{c}\text { Vertices } \\
\text { Visited } \\
\text { [Landmarks] }\end{array}$ & $\begin{array}{c}\text { Runtime } \\
\text { [Dijkstra] (s) }\end{array}$ & $\begin{array}{c}\text { Vertices } \\
\text { Visited } \\
\text { [Dijkstra] }\end{array}$ \\
\hline 10000 & 17509 & 0.0193 & 0.00475 & 439 & 0.0044 & 439 \\
\hline 11000 & 19344 & 0.0208 & 0.00505 & 416 & 0.00445 & 434 \\
\hline 12000 & 21166 & 0.0226 & 0.00555 & 488 & 0.00515 & 488 \\
\hline 13000 & 22999 & 0.0248 & 0.0058 & 494 & 0.0053 & 493 \\
\hline 14000 & 24869 & 0.0269 & 0.0063 & 540 & 0.00565 & 548 \\
\hline 15000 & 26698 & 0.0282 & 0.0068 & 536 & 0.00635 & 550 \\
\hline
\end{tabular}


International Journal of Computer Science \& Information Technology (IJCSIT), Vol 3, No 1, Feb 2011

\begin{tabular}{|r|r|r|r|r|r|r|}
\hline 16000 & 28555 & 0.0304 & 0.00725 & 554 & 0.00655 & 554 \\
\hline 17000 & 30399 & 0.0358 & 0.00945 & 656 & 0.0087 & 656 \\
\hline 18000 & 32251 & 0.0383 & 0.00955 & 580 & 0.00905 & 594 \\
\hline 19000 & 34111 & 0.0412 & 0.01 & 652 & 0.0093 & 679 \\
\hline 20000 & 35966 & 0.0452 & 0.0112 & 648 & 0.0102 & 666 \\
\hline 21000 & 37844 & 0.0479 & 0.0113 & 683 & 0.0109 & 626 \\
\hline 22000 & 39705 & 0.0518 & 0.0127 & 675 & 0.0112 & 718 \\
\hline 23000 & 41550 & 0.0578 & 0.0139 & 801 & 0.0146 & 828 \\
\hline 24000 & 43435 & 0.0603 & 0.0144 & 767 & 0.0129 & 766 \\
\hline 25000 & 45286 & 0.0655 & 0.0144 & 794 & 0.0136 & 794 \\
\hline
\end{tabular}

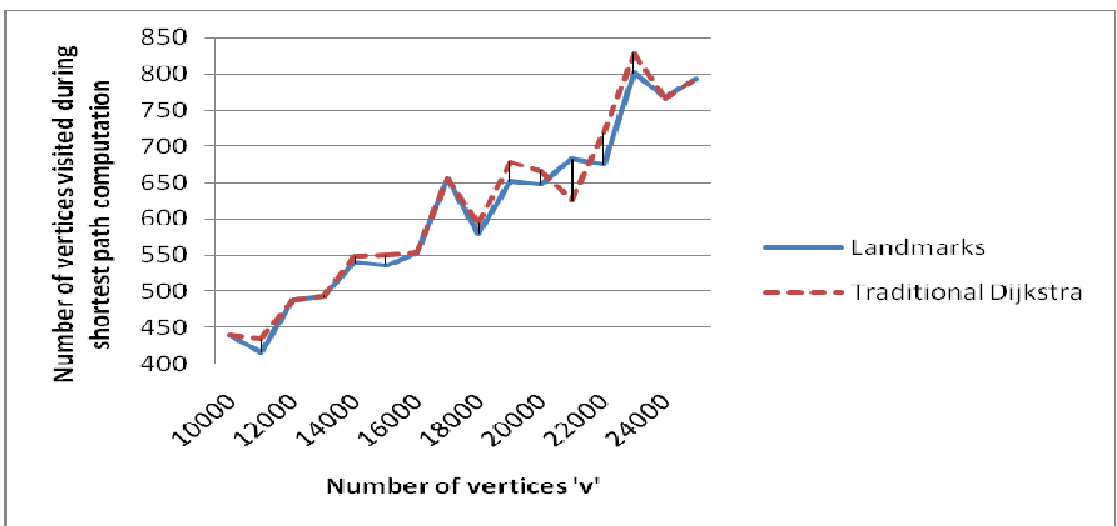

Figure 4. Vertices visited during shortest path computation by traditional Dijkstra and search procedure with Landmarks on planar graphs

\subsection{PERFORMANCE OF GEOMETRIC CONTAINERS ON RANDOM GRAPHS}

Table 3. Runtime and visited vertices comparison of Geometric containers and traditional Dijkstra on random graphs

\begin{tabular}{|r|r|r|r|r|r|r|}
\hline $\begin{array}{r}\text { Vertex } \\
\text { Count }\end{array}$ & $\begin{array}{c}\text { Edge } \\
\text { Count }\end{array}$ & $\begin{array}{c}\text { Preprocessing } \\
\text { Time (s) }\end{array}$ & $\begin{array}{c}\text { Runtime [with } \\
\text { Containers] (s) }\end{array}$ & $\begin{array}{c}\text { Vertices Visited } \\
\text { [Containers] }\end{array}$ & $\begin{array}{c}\text { Runtime } \\
\text { [Dijkstra] (s) }\end{array}$ & $\begin{array}{c}\text { Vertices } \\
\text { Visited } \\
\text { [Dijkstra] }\end{array}$ \\
\hline 1000 & 8000 & 1.879 & 0.0013 & 512 & 0.001 & 325 \\
\hline 2000 & 16000 & 7.99 & 0.0026 & 984 & 0.0028 & 1204 \\
\hline 3000 & 21000 & 17.9 & 0.0042 & 1430 & 0.0038 & 1592 \\
\hline 4000 & 20000 & 27.9 & 0.0037 & 1316 & 0.0048 & 2306 \\
\hline 5000 & 25000 & 47.1 & 0.006 & 1778 & 0.006 & 2749 \\
\hline 6000 & 30001 & 73.5 & 0.0086 & 2749 & 0.007 & 3000 \\
\hline 7000 & 63000 & 148 & 0.0138 & 3233 & 0.0108 & 2959 \\
\hline 8000 & 72000 & 214 & 0.0128 & 2640 & 0.0139 & 3596 \\
\hline 9000 & 72000 & 257 & 0.0158 & 3224 & 0.0182 & 5373 \\
\hline 10000 & 100000 & 389 & 0.029 & 5634 & 0.0229 & 5312 \\
\hline
\end{tabular}

Table 3. shows the experimental values obtained by comparing Geometric Containers with the traditional shortest path computation technique. A speedup of 1.2 is achieved based on the number of vertices visited during the shortest path computation. The average running time of the search with geometric containers nearly equals that of traditional search. Two important points of interest are as follows. The first one is that increasing the number of vertices can reduce the running time but due the memory limitations of the experimental setup and the 
libraries used the vertex count was not increased during the analysis. The second point to note is that geometric containers have a better performance in real word graphs and this was not tested due to time limitations.

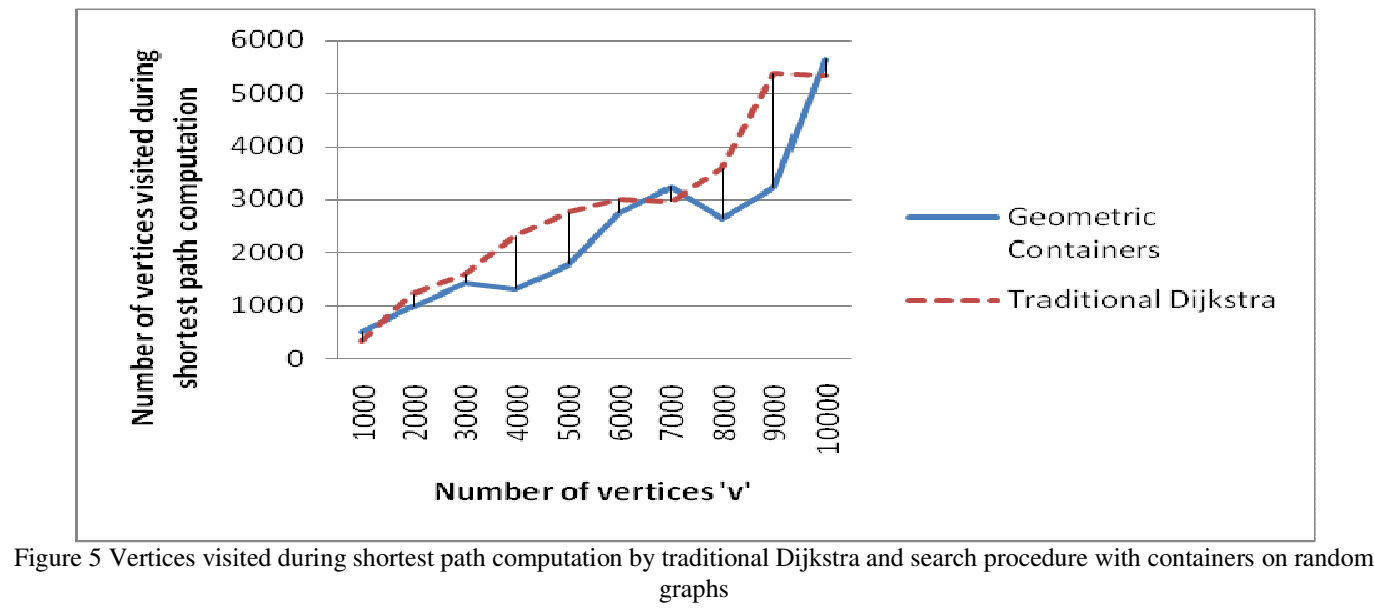

Figure 5. shows the number of vertices visited by traditional search technique and the search with containers, plotted against the number of vertices present in the graph.

\subsection{USING GEOMETRIC CONTAINERS ON PLANAR GRAPHS}

Using geometric containers on planar graphs generated by LEDA had a meagre performance on the number of vertices visited. The values are shown in Table 4. It gives a varying results of running time and vertices visited compared to that of Dijkstra.

Table 4. Runtime and visited vertices comparison of Geometric containers and traditional Dijkstra on planar graphs

\begin{tabular}{|r|r|r|r|r|r|r|}
\hline $\begin{array}{c}\text { Vertex } \\
\text { Count }\end{array}$ & $\begin{array}{c}\text { Edge } \\
\text { Count }\end{array}$ & $\begin{array}{c}\text { Preprocessing } \\
\text { Time (s) }\end{array}$ & $\begin{array}{c}\text { Runtime [with } \\
\text { Containers] (s) }\end{array}$ & $\begin{array}{c}\text { Vertices } \\
\text { Visited } \\
\text { [Containers] }\end{array}$ & $\begin{array}{c}\text { Runtime } \\
\text { [Dijkstra] (s) }\end{array}$ & $\begin{array}{c}\text { Vertices } \\
\text { Visited } \\
\text { [Dijkstra] }\end{array}$ \\
\hline 10000 & 17470 & 25.085 & 0.0031 & 116 & 0.0047 & 106 \\
\hline 11000 & 19294 & 31.3 & 0.0015 & 78 & 0.0063 & 122 \\
\hline 12000 & 21102 & 36.9 & 0.0045 & 76 & 0.0048 & 103 \\
\hline 13000 & 23010 & 43.6 & 0.0047 & 99 & 0.0047 & 123 \\
\hline 14000 & 24857 & 50.5 & 0.0045 & 82 & 0.0048 & 156 \\
\hline 15000 & 26756 & 58.3 & 0.0063 & 119 & 0.0046 & 60 \\
\hline 16000 & 28556 & 66.2 & 0.0046 & 164 & 0.0063 & 46 \\
\hline 17000 & 30407 & 78 & 0.0125 & 160 & 0.0031 & 108 \\
\hline 18000 & 32180 & 87.6 & 0.014 & 118 & 0.0016 & 98 \\
\hline 19000 & 34164 & 98.1 & 0 & 112 & 0.0156 & 162 \\
\hline 20000 & 35953 & 109 & 0.0156 & 59 & 0 & 114 \\
\hline
\end{tabular}

\subsection{COMBINATION OF LANDMARKS AND GEOMETRIC CONTAINERS APPLIED TO RANDOM GRAPHS}

The important technique implemented in this work is a search procedure that combines both the landmarks and geometric containers during shortest path computation. The graphs considered for analysis by this technique are undirected, though it is possible to apply the 
technique to directed-graphs, slight changes to the landmarks module will be necessary. Table 5. shows the experimental results. Though the pre-processing time is high, the process occurs only once and therefore excluded from the shortest path computation runtime values. The new technique reduces the number of vertices visited during the shortest path query evaluation and speedup of 1.79 based on vertex-visit-count is achieved. The values in table 5. are with respect to random graphs generated by LEDA.

Table 5. Combination of Landmarks and Geometric Containers compared with traditional Dijkstra on Random Graphs

\begin{tabular}{|c|c|c|c|c|c|c|c|}
\hline $\begin{array}{c}\text { Vertex } \\
\text { Count }\end{array}$ & $\begin{array}{c}\text { Edge } \\
\text { Count }\end{array}$ & $\begin{array}{c}\text { Preprocess } \\
\text { Time } \\
\text { [Landmarks] }\end{array}$ & $\begin{array}{c}\text { Runtime } \\
\text { [Combination] } \\
(\mathbf{s})\end{array}$ & $\begin{array}{c}\text { Vertices } \\
\text { Visited } \\
\text { [Combination] }\end{array}$ & $\begin{array}{c}\text { Runtime } \\
\text { [Dijkstra] } \\
\text { (s) }\end{array}$ & $\begin{array}{c}\text { Vertices } \\
\text { Visited } \\
\text { [Dijkstra] }\end{array}$ & $\begin{array}{c}\text { Preprocess } \\
\text { Time } \\
\text { [Containers] }\end{array}$ \\
\hline 1000 & 10000 & 0.019 & 0.001 & 85 & 0.0015 & 276 & 3.13 \\
\hline 2000 & 20000 & 0.0325 & 0.0055 & 552 & 0.003 & 348 & 13.8 \\
\hline 3000 & 30000 & 0.0725 & 0.007 & 579 & 0.011 & 2597 & 38.1 \\
\hline 4000 & 40000 & 0.099 & 0.022 & 1801 & 0.014 & 2699 & 80.3 \\
\hline 5000 & 50000 & 0.139 & 0.027 & 1993 & 0.0195 & 3040 & 145 \\
\hline 6000 & 60000 & 0.227 & 0.0155 & 892 & 0.013 & 1495 & 229 \\
\hline 7000 & 70000 & 0.237 & 0.0325 & 1884 & 0.038 & 5501 & 339 \\
\hline 8000 & 80000 & 0.348 & 0.0244 & 1228 & 0.0355 & 4868 & 467 \\
\hline 9000 & 90000 & 0.347 & 0.0405 & 2760 & 0.0375 & 3067 & 633 \\
\hline 10000 & 100000 & 0.296 & 0.0386 & 2062 & 0.0080 & 944 & 781 \\
\hline
\end{tabular}

\subsection{COMBINATION OF LANDMARKS AND GEOMETRIC CONTAINERS APPLIED TO} PLANAR GRAPHS

Table 6. Combination of Landmarks and Geometric Containers compared with traditional Dijkstra on Planar Graphs

\begin{tabular}{|c|c|c|c|c|c|c|c|}
\hline $\begin{array}{l}\text { Vertex } \\
\text { Count }\end{array}$ & $\begin{array}{l}\text { Edge } \\
\text { Count }\end{array}$ & $\begin{array}{c}\text { Preprocess } \\
\text { Time } \\
\text { [Landmarks] }\end{array}$ & $\begin{array}{c}\text { Runtime } \\
\text { [Combination] } \\
(\mathbf{s})\end{array}$ & $\begin{array}{c}\text { Vertices } \\
\text { Visited } \\
\text { [Combination] }\end{array}$ & $\begin{array}{c}\text { Runtime } \\
\text { [Dijkstra] } \\
\text { (s) }\end{array}$ & $\begin{array}{c}\text { Vertices } \\
\text { Visited } \\
\text { [Dijkstra] }\end{array}$ & $\begin{array}{c}\text { Preprocess } \\
\text { Time } \\
\text { [Containers] }\end{array}$ \\
\hline 1000 & 1519 & 0.0025 & 0 & 40 & 0.001 & 124 & 0.256 \\
\hline 2000 & 3213 & 0.0035 & 0.0005 & 71 & 0.001 & 123 & 1 \\
\hline 3000 & 4932 & 0.005 & 0.000999 & 119 & 0.001 & 184 & 2.26 \\
\hline 4000 & 6659 & 0.007 & 0.001 & 69 & 0.002 & 226 & 4.01 \\
\hline 5000 & 8435 & 0.009 & 0.002 & 74 & 0.002 & 199 & 6.26 \\
\hline 6000 & 10249 & 0.011 & 0.002 & 133 & 0.003 & 298 & 9.05 \\
\hline 7000 & 12075 & 0.012 & 0.003 & 361 & 0.003 & 445 & 12.4 \\
\hline 8000 & 13878 & 0.013 & 0.0035 & 310 & 0.003 & 437 & 16.3 \\
\hline 9000 & 15696 & 0.017 & 0.0035 & 234 & 0.004 & 349 & 20.7 \\
\hline 10000 & 17512 & 0.0185 & 0.005 & 356 & 0.004 & 650 & 25.6 \\
\hline
\end{tabular}

Table 6. shows the experimental values obtained by testing the combined speedup technique, combining landmarks with containers, on planar graphs generated by LEDA.

A speedup of 1.12 was achieved based on the running time of the technique whereas the speedup was 1.7 with respect the number of vertices visited during the shortest path computation.

Figure 6. compares the running time of the combined speedup technique with that of Dijkstra's and the average running time is observed to be slightly improved. As mentioned 
earlier containers perform well when applied to real world graphs and hence the combined speedup technique is also expected to perform better in such a scenario.

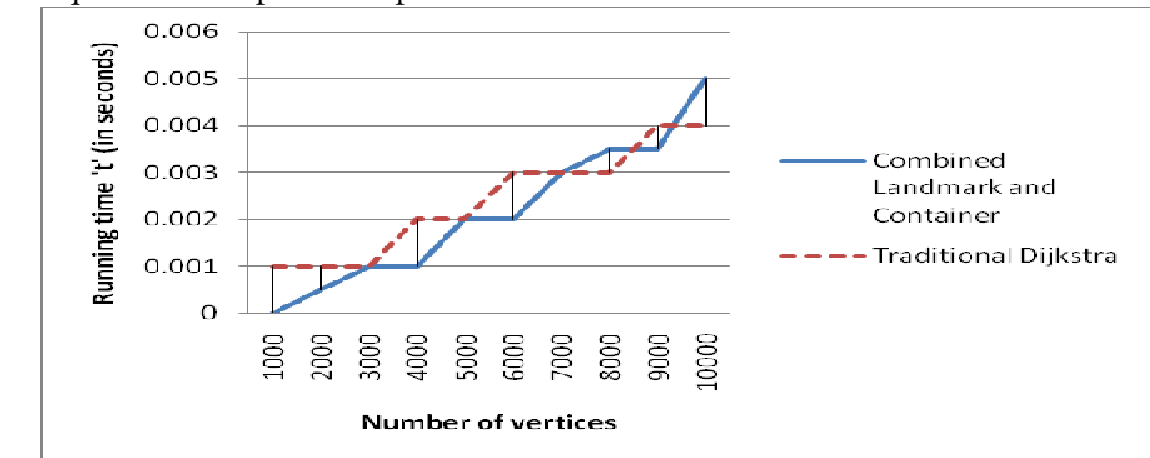

Figure 6. Running time of the "combined landmark and container" speedup technique compared with Dijkstra's algorithm

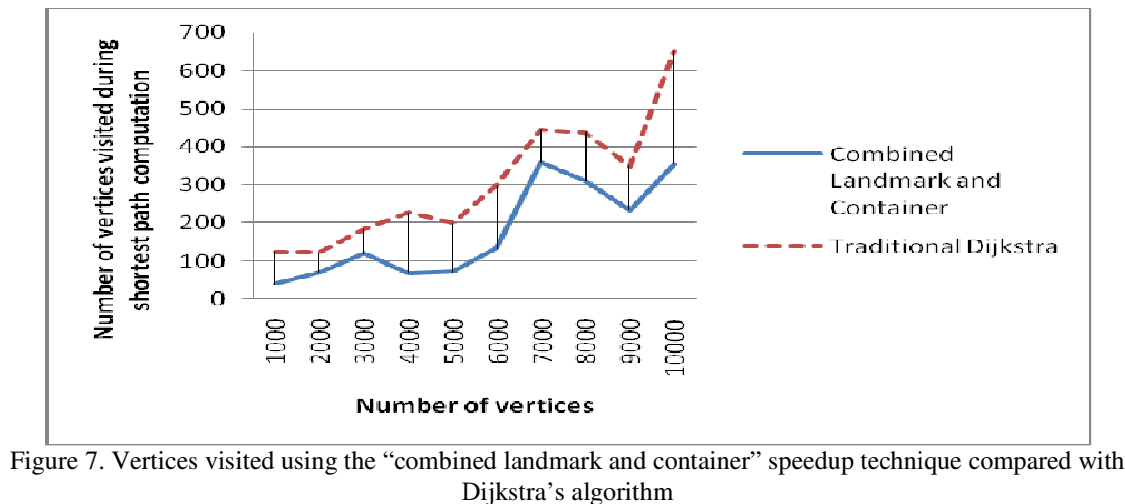

Figure 7. is the graphical representation of the number of vertices visited by the techniques under comparison, viz., "combined landmarks and containers" and traditional Dijkstra's algorithm.

From the tabulated values it can be inferred that the combined speedup technique improves the performance of shortest path computation to a considerable extent.

\subsection{PARALlELISING THE PREPROCESSING PHASE OF LANDMARKS (RANDOM GRAPHS)}

Table7 compares the running time of the preprocessing phase of landmarks with and without parallelism. A speedup of 1.13 was achieved on the random graphs generated by LEDA. Figure 8 presents a graphical representation of the running time of the preprocessing phase of landmarks before and after parallelism is incorporated.

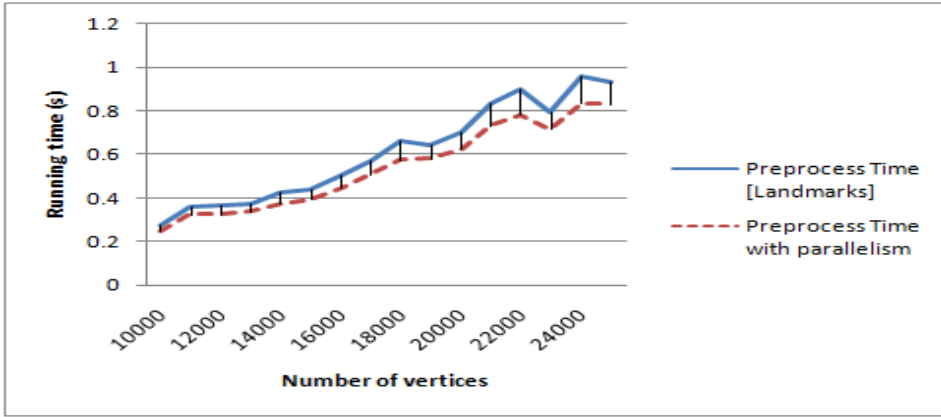

Figure 8. Comparison of Preprocessing Time for Landmarks with and without Parallelism on random graphs 
International Journal of Computer Science \& Information Technology (IJCSIT), Vol 3, No 1, Feb 2011

Table 7. Analysis of Parallelised Preprocessing in Landmarks (random graphs)

\begin{tabular}{|c|c|c|c|}
\hline Vertex Count & Edge Count & $\begin{array}{c}\text { Preprocess Time } \\
\text { [Landmarks] (s) }\end{array}$ & $\begin{array}{c}\text { Preprocess Time with } \\
\text { parallelism (s) }\end{array}$ \\
\hline 10000 & 78000 & 0.274 & 0.246 \\
\hline 11000 & 82500 & 0.361 & 0.323 \\
\hline 12000 & 90600 & 0.368 & 0.324 \\
\hline 13000 & 97500 & 0.373 & 0.335 \\
\hline 14000 & 103600 & 0.424 & 0.374 \\
\hline 15000 & 108000 & 0.44 & 0.393 \\
\hline 16000 & 104000 & 0.507 & 0.443 \\
\hline 17000 & 127500 & 0.569 & 0.507 \\
\hline 18000 & 128700 & 0.663 & 0.573 \\
\hline 19000 & 138700 & 0.644 & 0.579 \\
\hline 20000 & 141000 & 0.704 & 0.622 \\
\hline 21000 & 162750 & 0.833 & 0.73 \\
\hline 22000 & 157300 & 0.902 & 0.779 \\
\hline 23000 & 167900 & 0.794 & 0.715 \\
\hline 24000 & 175200 & 0.961 & 0.832 \\
\hline 25000 & 178750 & 0.932 & 0.83 \\
\hline
\end{tabular}

\subsection{PARALLELISING THE PREPROCESSING PHASE OF LANDMARKS (PLANAR GRAPHS)}

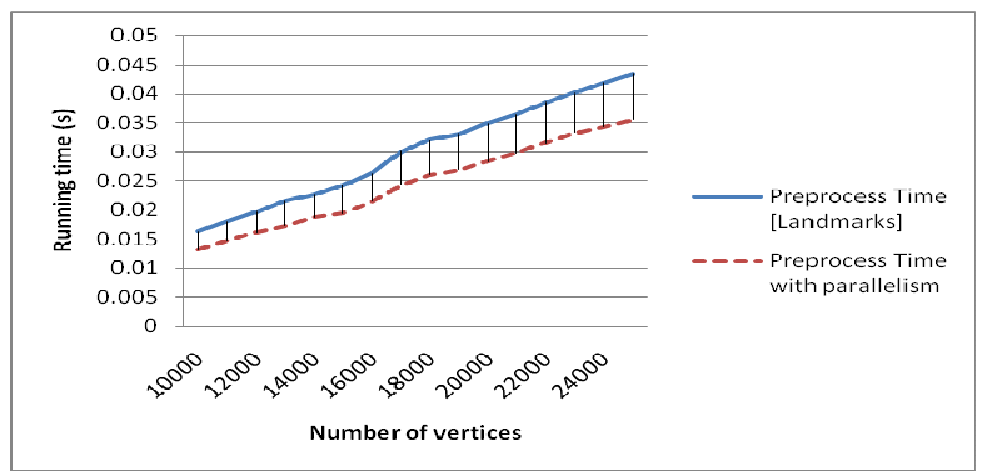

Figure 9. Comparison of Preprocessing Time for Landmarks with and without Parallelism on planar graphs

Table 8. Analysis of Parallelised Preprocessing in Landmarks (Planar graphs)

\begin{tabular}{|c|c|c|c|}
\hline $\begin{array}{c}\text { Vertex } \\
\text { Count }\end{array}$ & Edge Count & $\begin{array}{c}\text { Preprocess Time } \\
\text { [Landmarks] (s) }\end{array}$ & $\begin{array}{c}\text { Preprocess Time with parallelism } \\
\text { (s) }\end{array}$ \\
\hline 10000 & 17516 & 0.0163 & 0.0132 \\
\hline 11000 & 19342 & 0.018 & 0.0147 \\
\hline 12000 & 21190 & 0.0197 & 0.0161 \\
\hline 13000 & 23018 & 0.0215 & 0.0173 \\
\hline 14000 & 24856 & 0.0227 & 0.0188 \\
\hline 15000 & 26685 & 0.0243 & 0.0195 \\
\hline
\end{tabular}


International Journal of Computer Science \& Information Technology (IJCSIT), Vol 3, No 1, Feb 2011

\begin{tabular}{|l|l|l|l|}
\hline 16000 & 28561 & 0.0263 & 0.0215 \\
\hline 17000 & 30400 & 0.0301 & 0.0243 \\
\hline 18000 & 32246 & 0.0321 & 0.0261 \\
\hline 19000 & 34118 & 0.0331 & 0.0269 \\
\hline 20000 & 35954 & 0.0349 & 0.0284 \\
\hline 21000 & 37813 & 0.0365 & 0.0298 \\
\hline 22000 & 39705 & 0.0385 & 0.0316 \\
\hline 23000 & 41570 & 0.0402 & 0.0332 \\
\hline 24000 & 43437 & 0.0418 & 0.0343 \\
\hline 25000 & 45308 & 0.0434 & 0.0355 \\
\hline
\end{tabular}

Table 8 compares the preprocessing phase running time for landmarks with and without parallelism. A speedup of 1.22 was achieved on the planar graphs generated by LEDA.

Figure 9 plots the preprocessing time required for landmarks with and without parallelism against the number of vertices.

\subsection{PARALLELISING PREPROCESSING IN THE COMBINED SPEEDUP TECHNIQUE (RANDOM GRAPHS)}

Table 9. Analysis of Parallelised Preprocessing in the Combined speedup Technique (random graphs)

\begin{tabular}{|c|c|c|c|c|c|}
\hline $\begin{array}{c}\text { Vertex } \\
\text { Count }\end{array}$ & Edge Count & $\begin{array}{c}\text { Preprocess } \\
\text { Time (s) }\end{array}$ & $\begin{array}{c}\text { Preprocess } \\
\text { Time with } \\
\text { parallelism (s) }\end{array}$ & $\begin{array}{c}\text { Preprocess } \\
\text { Time } \\
\text { [Landmarks } \\
\text { section] (s) }\end{array}$ & $\begin{array}{c}\text { Preprocess Time with } \\
\text { parallelism [Landmarks } \\
\text { section] (s) }\end{array}$ \\
\hline 1000 & 7500 & 2.5040 & 2.5040 & 0.0000 & 0.0000 \\
\hline 2000 & 17000 & 11.7240 & 11.7240 & 0.0160 & 0.0000 \\
\hline 3000 & 25500 & 29.8040 & 29.7880 & 0.0300 & 0.0320 \\
\hline 4000 & 26000 & 45.5450 & 45.5360 & 0.0470 & 0.0160 \\
\hline 5000 & 32500 & 85.3480 & 85.3170 & 0.0780 & 0.0000 \\
\hline 6000 & 30000 & 107.2600 & 107.2400 & 0.0640 & 0.0000 \\
\hline 7000 & 42000 & 192.7800 & 192.7000 & 0.0930 & 0.0000 \\
\hline 8000 & 56000 & 317.6500 & 317.5700 & 0.2030 & 0.0930 \\
\hline 9000 & 76500 & 548.2100 & 548.1300 & 0.2810 & 0.1410 \\
\hline 10000 & 100000 & 808.9800 & 808.8500 & 0.2960 & 0.1560 \\
\hline
\end{tabular}

Table 9 shows the preprocessing time of the combined speedup technique, with parallelism incorporated appropriately. It is noted that the preprocessing time is reduced only by a marginal value with parallelism included; the reason for this is that, the container construction module takes the maximum of preprocessing time and it remains sequential. Only preprocessing related to landmarks is parallelised; the corresponding values are also separately included in the table.

\subsection{PARALLELISING PREPROCESSING IN THE COMBINED SPEEDUP TECHNIQUE (PLANAR GRAPHS)}


International Journal of Computer Science \& Information Technology (IJCSIT), Vol 3, No 1, Feb 2011

Table 10. Analysis of Parallelised Preprocessing in the Combined speedup Technique (Planar Graphs)

\begin{tabular}{|c|c|c|c|c|c|}
\hline $\begin{array}{c}\text { Vertex } \\
\text { Count }\end{array}$ & Edge Count & $\begin{array}{c}\text { Preprocess } \\
\text { Time (s) }\end{array}$ & $\begin{array}{c}\text { Preprocess Time } \\
\text { with parallelism } \\
\text { (s) }\end{array}$ & $\begin{array}{c}\text { Preprocess Time } \\
\text { [Landmark } \\
\text { section] (s) }\end{array}$ & $\begin{array}{c}\text { Preprocess Time } \\
\text { with parallelism } \\
\text { [Landmark } \\
\text { section] (s) }\end{array}$ \\
\hline 1000 & 1500 & 0.2545 & 0.2540 & 0.0015 & 0.0010 \\
\hline 2000 & 3203 & 0.9950 & 0.9945 & 0.0015 & 0.0010 \\
\hline 3000 & 4938 & 2.2390 & 2.2370 & 0.0030 & 0.0010 \\
\hline 4000 & 6677 & 3.9815 & 3.9795 & 0.0030 & 0.0010 \\
\hline 5000 & 8431 & 6.2065 & 6.2040 & 0.0040 & 0.0015 \\
\hline 6000 & 10295 & 9.0320 & 9.0290 & 0.0050 & 0.0020 \\
\hline 7000 & 12034 & 12.3580 & 12.3540 & 0.0060 & 0.0020 \\
\hline 8000 & 13876 & 16.2770 & 16.2710 & 0.0075 & 0.0020 \\
\hline 9000 & 15702 & 20.6960 & 20.6920 & 0.0075 & 0.0030 \\
\hline 10000 & 17493 & 25.5750 & 25.5720 & 0.0085 & 0.0050 \\
\hline
\end{tabular}

Table 10 presents the preprocessing time variations of the combined speedup technique on planar graphs with suitable portions parallelised. The improvement achieved is less.

\section{CONCLUSION}

The speedup techniques for Dijkstra's algorithm like Landmarks and Geometric containers were analysed with random graphs and planar graphs. Important metrics for evaluation of the techniques like speedup based on running time and the number of vertices visited during shortest path computation were considered. The technique of combining landmarks and geometric containers was also analysed for the same graph types.

Each speedup technique worked well for a specific type of graph and hence the performance was appreciable in those cases. The heuristic values obtained by using landmarks helped to reduce the number of vertices visited during shortest path computation but the running time of the technique was marginally high due the computation overhead involved during vertex distance updation process. The geometric containers achieved speedup based on the vertices visited during the evaluation of shortest path query but were nearly equal in running time to the traditional search process.

The combined speed up technique based on landmarks and containers was able to perform better under the same experimental setup compared to the other techniques. Based on the running time the speedup was 1.12 while based on the number of vertices visited the speedup attained was 1.7 .

The performance is expected to be improved on real world graphs compared to the graphs generated by LEDA.

\section{REFERENCES}

[1] DIJKSTRA, E. W, (1959) "A note on two problems in connection with graphs", In Numerische Mathematik, Vol. 1, Mathematisch Centrum, Amsterdam, The Netherlands, pp269-271.

[2] Andrew V.Goldberg \& Chris Harrelson, (2005) "Computing the Shortest Path: A* Search Meets Graph Theory", In Proc. $16^{\text {th }}$ Annual ACM-SIAM Symposium on Discrete Algorithms.

[3] I. Phol, (1971) "Bi-directional Search", In Machine Intelligence, volume 6, pp 124-140. Edinburgh Univ. Press, Edinburgh.

[4] Martin Holzer, (2003) "Hierarchical speedup techniques for shortest path algorithms", M, Tech. report, Dept of Informatics, University of Konstanz, Germany. 
[5] Frank Schulz, Dorothea Wagner, \& Christos Zaroliagis, (2002) "Using multi-level graphs for timetable information in railway systems", In Proc. $4^{\text {th }}$ Workshop on Algorithm Engineering and Experiments. LNCS 2409, Springer-Verlag, New York. pp43- 59.

[6] Dorothea Wagner, Thomas Willhalm, \& Christos Zaroliagis, (2005) "Geometric Containers for Efficient Shortest-Path Computation”, ACM Journal of Experimental Algorithmics, 10(1.3).

[7] Martin Holzer, Frank Schulz, Dorothea Wagner, \& Thomas Willhalm, (2005) "Combining Speed-up Techniques for Shortest-Path Computations", ACM Journal of Experimental Algorithmics, Vol. 10, Article No. 2.5.

[8] Reinhard Bauer, Daniel Delling, Peter Sanders, Dennis Schieferdecker, Dominik Schultes, \& Dorothea Wagner, (2010) "Combining hierarchical and goal-directed speed-up techniques for dijkstra's algorithm”, ACM Journal of Experimental Algorithmics, Vol. 15, Article No. 3.

[9] Bauer. R, Delling. D, Sanders. P, Schieferdecker. D, Schultes. D \& Wagner. D, (2008) "Combining hierarchical and goal-directed speed-up techniques for dijkstra's algorithm", in the proceedings of the $7^{\text {th }}$ Workshop on Experimental Algorithms(WEA'08), Springer, Berlin, pp.303-318.

[10] Dominik Schultes, Johannes Singler, \& Peter Sanders, (2008) "Parallel Highway Node Routing", A Technical Report. algo2.iti.kit.edu/schultes/hwy/parallelHNR.pdf

[11] OpenMP, http://www.openmp.org

[12] LEDA, http://www.algorithmic-solutions.com

\section{Authors}

R. Kalpana is working as Associate Professor in the Department of Computer Science \& Engineering, Pondicherry Engineering College, Pondicherry, India.She has completed her B.Tech(Computer Science \& Engineering) from Pondicherry University.She has completed her M.Tech(Computer Science \& Engineering) from Pondicherry University. She is pursuing Ph.D at Pondicherry University. She has published ten papers in national and international conferences/ journals.

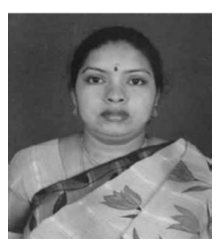

Prof. Dr. P. Thambidurai is the Principal and Professor of Computer Science \& Engineering, Perunthalaivar Kamarajar College of Engineering and Technology, Karaikal. He has completed his M.E(Computer Science \& Engineering) from Anna University, Chennai. He has completed his Ph.D from Alagappa University, Karaikudi, Tamilnadu. He has published more than hundred papers in national, International journals and conferences.

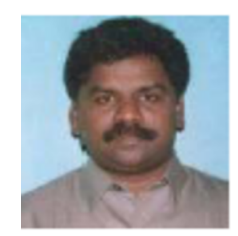

